\title{
THE DETECTION OF NUMEROUS MAGNETIC SEPARATORS IN A THREE-DIMENSIONAL MAGNETOHYDRODYNAMIC MODEL OF SOLAR EMERGING FLUX
}

\author{
C. E. Parnell, R. C. Maclean, and A. L. Haynes \\ School of Mathematics and Statistics, University of St Andrews, The North Haugh, St Andrews, Fife, KY16 9SS, UK; clare@mcs.st-and.ac.uk \\ Received 2010 March 18; accepted 2010 November 15; published 2010 December 6
}

\begin{abstract}
Magnetic separators in three-dimensional (3D) magnetic fields are believed to be often associated with locations of magnetic reconnection. In this preliminary study, we investigate this relationship using data from a numerical resistive 3D MHD experiment of a solar flux emergence event. For the first time separators are detected in complex magnetic fields resulting from a 3D resistive MHD model of flux emergence. Two snapshots of the model, taken from different stages of its evolution, are analyzed. Numerous separators are found in both snapshots, and their properties, including their geometry, length, relationship to the magnetic null points, and integrated parallel electric field are studied. The separators reside at the junctions between the emerging flux, the overlying field, and two other flux domains that are newly formed by reconnection. The long separators, which connect clusters of nulls that lie either side of the emerging flux, pass through spatially localized regions of high parallel electric field and correspond to local maxima in integrated parallel electric field. These factors indicate that strong magnetic reconnection takes place along many of the separators, and that separators play a key role during the interaction of emerging and overlying flux.
\end{abstract}

Key words: magnetic fields - magnetic reconnection - methods: numerical - plasmas - Sun: atmosphere - Sun: corona

Online-only material: color figures

\section{INTRODUCTION}

Magnetic topological analysis is a powerful tool for understanding the three-dimensional (3D) structure, connectivity, and evolution of magnetic fields (e.g., see Longcope 2005). Until recently, the computational techniques and resources to perform full topological analysis of highly complex 3D magnetic fields have not existed. Now, however, Haynes \& Parnell (2010) have developed techniques to determine the presence and location of magnetic separators (and other topological features such as null points, separatrix surfaces, and spines) in complex 3D magnetic field data sets. Therefore, the results of theoretical magnetic topology can at last be applied to numerical experiments and so provide a greater understanding of the solar phenomena being modeled.

We applied these new techniques to calculate the locations of magnetic separators in a 3D MHD model of flux emergence on the Sun. The emergence of new magnetic flux on the Sun and its interaction with preexisting flux is a good test problem for these techniques because it involves strong magnetic reconnection (Priest \& Schrijver 2004) taking place within a magnetic field that has already been well studied (Archontis et al. 2004, 2005; Galsgaard et al. 2005, 2007), although its topological structure has not been calculated before. A topological analysis of the magnetic field involved in this process could shed more light on the location, nature, and rate of the magnetic reconnection, since magnetic separators are known to be good sites for magnetic reconnection (Sonnerup 1979; Lau \& Finn 1990; Longcope \& Cowley 1996; Galsgaard \& Nordlund 1997; Galsgaard et al. 2000; Longcope 2001; Pontin \& Craig 2006; Priest et al. 2005; Haynes et al. 2007; Parnell et al. 2008, 2010). It could also tell us about how much power this reconnection at topological features contributes to coronal heating.

In previous work (Maclean et al. 2009), we studied the flux emergence model of Archontis et al. (2004), with a particular focus on the magnetic null points in the magnetic field. Only weak signatures of reconnection were observed at these nulls, but preliminary results suggested that the separators might be more strongly involved in the reconnection process. This Letter studies the properties of the separators in two representative snapshots from the experiment and takes an initial look at the role of magnetic reconnection at the separators.

\section{MODEL}

The flux emergence model analyzed here was first presented by Archontis et al. (2004) and further analyzed by Archontis et al. (2005) and Galsgaard et al. (2005, 2007), where full details of its parameters can be found. In summary, the model consists of a stably stratified convection zone, a cool isothermal photosphere, a transition region with a steep temperature gradient, and a hot isothermal corona. An initially horizontal overlying magnetic field exists in the corona. A twisted magnetic flux tube is placed in the convection zone which rises up into the atmosphere and interacts (reconnects) with the overlying magnetic field. The Copenhagen Stagger Code (Nordlund \& Galsgaard 1997) is used to solve the 3D time-dependent resistive MHD equations. The box is periodic in the horizontal directions and closed at the top and bottom. The resolution is $160 \times 148 \times 218$ grid cells (stretched in $z$ ). Following Archontis et al. (2004), we normalize with respect to photospheric values such that a magnetic field unit is $B_{\mathrm{ph}}=1.3 \times 10^{3} \mathrm{G}$ and a length unit is $H_{\mathrm{ph}}=170 \mathrm{~km}$. In these units our numerical box covers $(-70,70)$ in $x,(-60,60)$ in $y$, and $(-22,71)$ in $z$. The sound speed in the photosphere is $c_{s}=6.8 \mathrm{~km} \mathrm{~s}^{-1}$ and so the sound travel time for one unit length is $\tau_{c s}=25 \mathrm{~s}$. The model covers a total of $149 \tau_{c s}$ before the rising reconnected flux tube approaches the top boundary and the evolution is stopped.

Magnetic separator field lines divide four topologically distinct flux domains (Green 1989). They often start and end at magnetic null points, which have been analyzed already for this model by Maclean et al. (2009). We have located the 


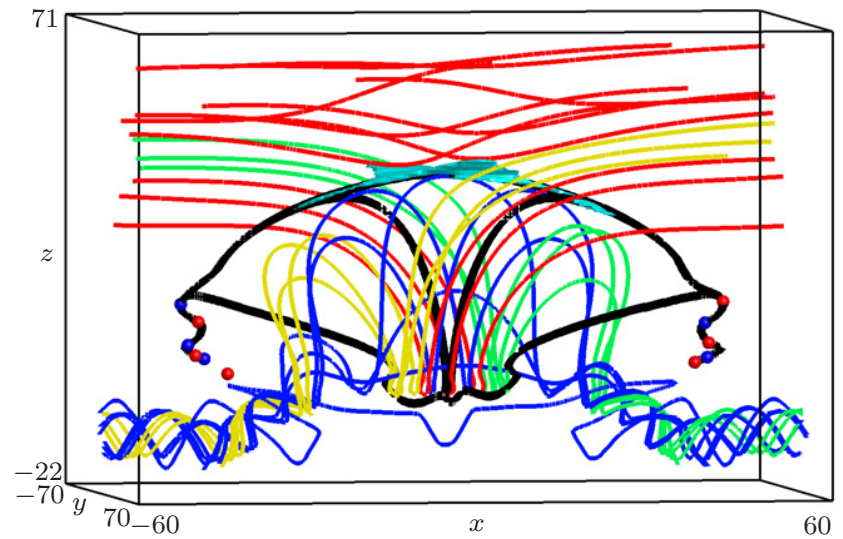

(a)
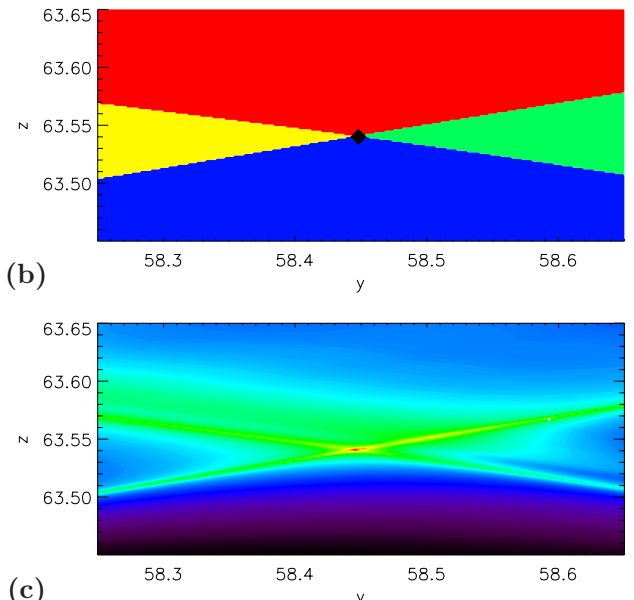





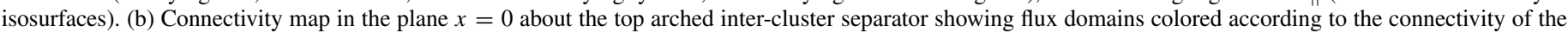

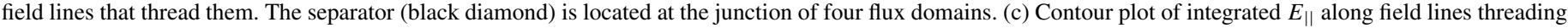
the same region. The top arched separator threads the plane at the location of the maximum integrated $E_{\|}$.

(A color version of this figure is available in the online journal.)

corresponding separators using the new separator-finding method of Haynes \& Parnell (2010). Briefly, this method works by first estimating the plane of the separatrix surface of each null by determining its eigenvalues and eigenvectors. A ring of points of small radius is then placed on the separatrix surface close to each null point. The rings are then repeatedly expanded outward by interpolation with successive steps producing new rings which map out the separatrix surface of that null. Once the outer ring of a separatrix surface meets a null of opposite polarity to its own null, then the ring is broken at the new null point and a separator is traced back through the points on the rings to the starting null point. This process is repeated for all the null points. All other approaches to finding separators use the connectivity of field lines, but these approaches are slow, inefficient, and sometimes ineffective for numerical magnetic field data sets, as discussed in Haynes \& Parnell (2010).

\section{ANALYSIS AND RESULTS}

We present an analysis of two snapshots from the model. One comes from the most complex phase of the interaction between the magnetic flux tube and the overlying field, at $86 \tau_{c s}$, and the other is taken from the later steady reconnection phase, after $109 \tau_{c s}$. Since the later snapshot (snapshot 109) has a much simpler structure than the earlier one (snapshot 86 ) we discuss this one first. In a later work, we will study the full time evolution of the model in more detail.

\subsection{Characteristics of the Separators in Snapshot 109}

Maclean et al. (2009) showed that the nulls form two loose clusters, one on each flank of the emerging flux tube and they speculated that these two clusters might be joined by separators. Using the detection algorithm of Haynes \& Parnell (2010), a total of 11 unique separators are found in snapshot 109, as shown in Figure 1(a). Within each null cluster, the separators join the nulls forming a simple chain, with three separators linking the two clusters. Each separator joins a pair of oppositely signed nulls, as expected, although of course the same pair of nulls may be joined by any number of separators (see Parnell 2007 for a discussion of such "multiply connected" nulls).
Of the twelve nulls in snapshot 109, two are isolated and are associated with no separators. The two nulls which have only one separator are at the ends of the chains in each cluster, while the six nulls which each have two separators lie in the chains themselves. Two further nulls each have four separators, three of which are inter-cluster separators. The inter-cluster separators all run from a positive null in the right-hand cluster to a negative one in the left-hand cluster (Figure 1(a)). One of the intercluster separators forms an arch between the null clusters and lies along the line in the corona that separates the emerging flux tube and overlying field. The central portions of the other two inter-cluster separators dip down below the photosphere and into the convection zone. A similar dipped structure for ordinary field lines was noted by Galsgaard et al. (2007) and can be seen in Figure 1(a).

Since the method we use to find the separators does not use connectivity, we confirm the existence of our separators by comparing them with connectivity maps. Figure 1(b) shows one such map about the top arched separator in the $x=0$ plane. The regions on the plane are colored according to the connectivity of the field lines that thread them and so these maps show a cut through the flux domains intersecting the plane. After reconnection has started four flux domains are present in the model: the original two (i.e., the emerging flux tube and overlying magnetic field) and two new domains formed by reconnection containing field lines that either start in the flux tube and end in the corona or vice versa. All four flux domains are present in Figure 1(b) and at their junction the top arched separator threads the plane as expected.

An electric field component parallel to the magnetic field is the signature of 3D magnetic reconnection (Hesse \& Schindler 1988). Moreover, a non-zero integral of parallel electric field $\left(E_{\|}\right)$along a field line is a necessary and sufficient condition for reconnection (Hesse \& Schindler 1988). For an isolated diffusion region the maximum integrated $E_{\|}\left(\int E_{\|} d l\right)$ is a measure of the reconnection rate in that diffusion region (Hesse \& Schindler 1988; Hornig \& Priest 2003; Parnell et al. 2008). So in order to verify the importance of the separators for reconnection, we calculate $\int E_{\|} d l$ along the separators and their surrounding field lines. As an example, the $\int E_{\|} d l$ along the 


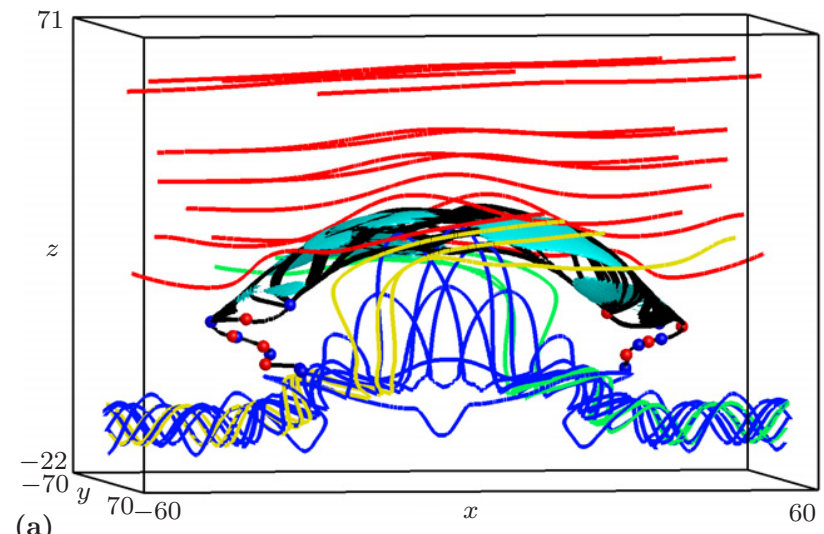

(a)

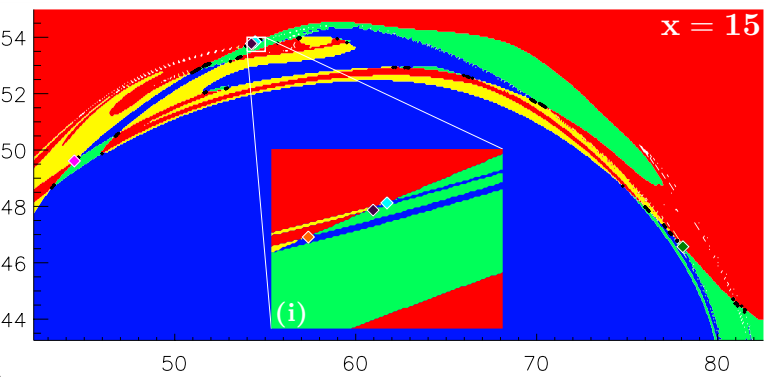

(b)
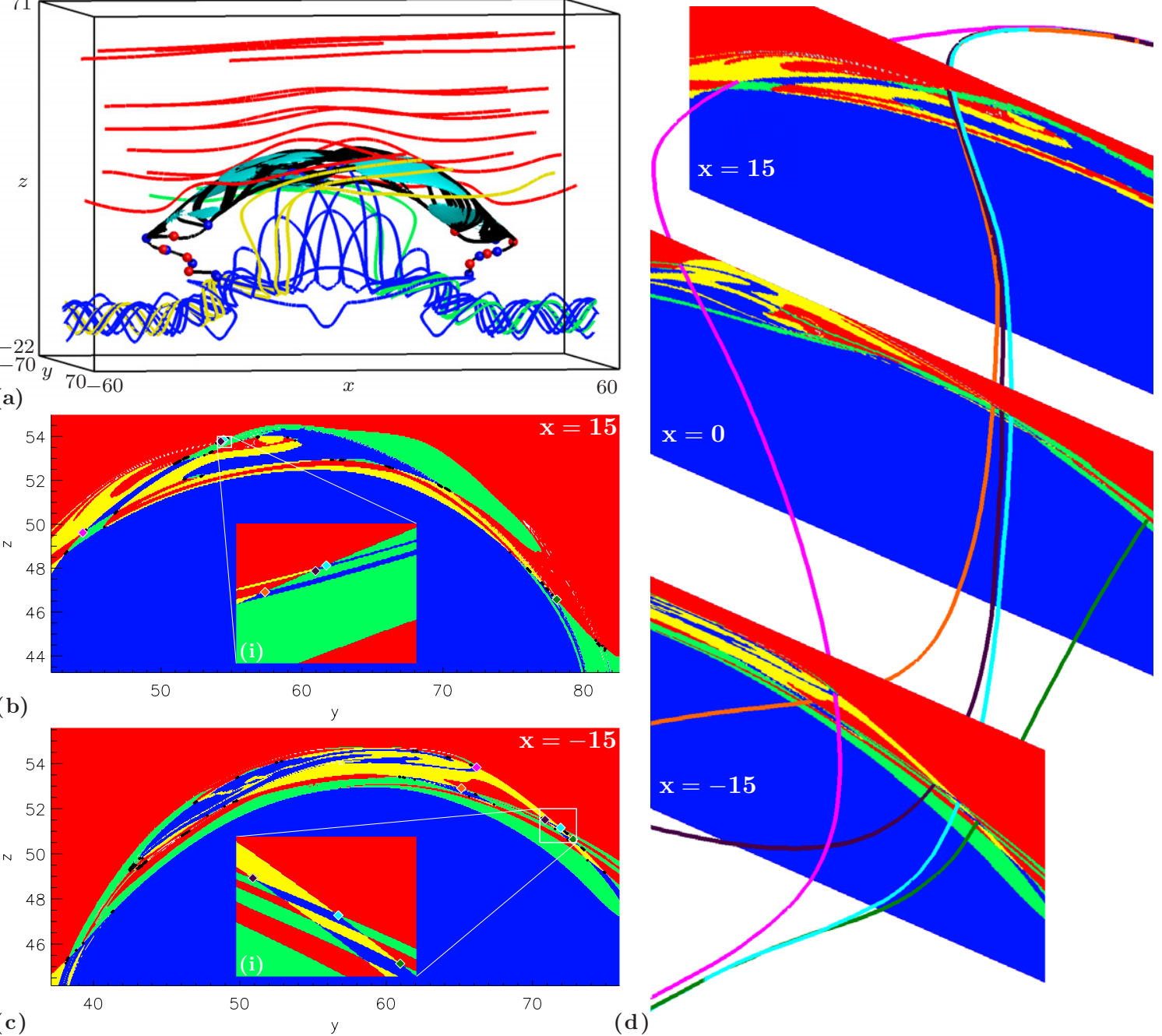

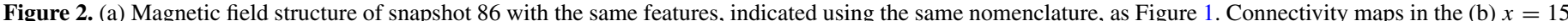

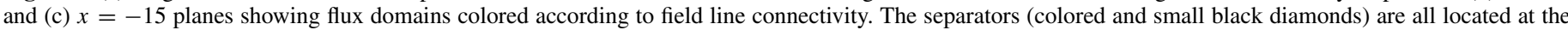

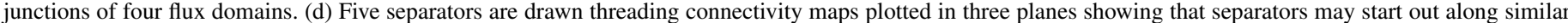

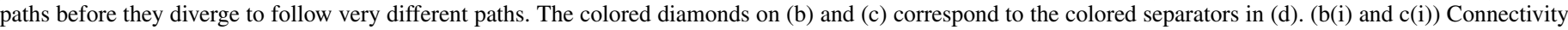
maps for the white boxed regions in (b) and (c), respectively.

(A color version of this figure is available in the online journal.)

field lines that thread the region about the top arched separator is plotted in Figure 1(c). The local maxima in $\int E_{\|} d l$ coincides with the separator, as it does for all the separators, indicating that the separators are the most important locations of reconnection in the model.

\subsection{Characteristics of the Separators in Snapshot 86}

Earlier on in the model the interaction of the emerging flux tube with the overlying field is much more complex and involves a total of 229 separators (Figure 2). As above, within each null cluster, the separators join the nulls forming a simple chain, but between the two clusters runs a highly complex arch-shaped mesh of separators (Figure 3).

Of the eighteen nulls in snapshot 86 , only one is completely isolated and has no separators (Figure 2(a)). Two nulls are associated with only one separator; these are the endpoints of the chain of nulls within each cluster. Eleven nulls each have two separators; these lie within the chains in the clusters. The remaining four null points have about 100 separators each $(98,119,103$, and 114, respectively, Figure 2). These are the

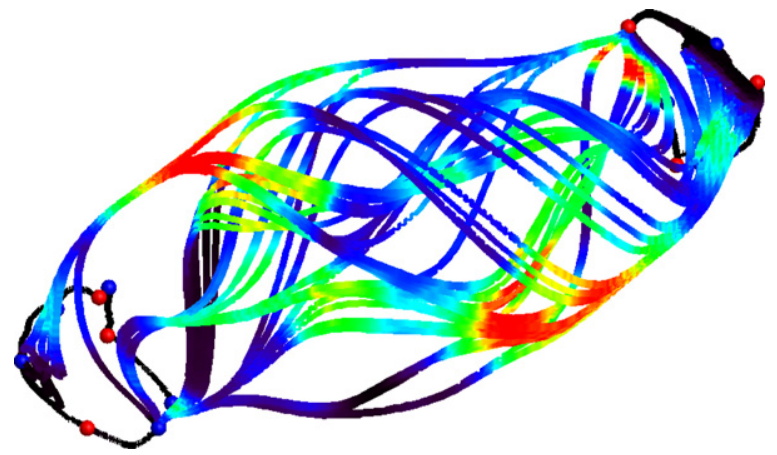

Figure 3. View from above of the separators in snapshot 86 which have been colored with $E_{\|}$(black: low and red: high).

(A color version of this figure is available in the online journal.)

four crucial nulls whose associated separators form the arch structure joining the two null clusters. All of the 214 inter-cluster separators run in the same direction: from one of two positive nulls in the right-hand cluster to either of the two negative nulls in the left-hand cluster (Figure 2(a)). The separators often start 
off and run along very similar paths for much of their lengths before diverging and following the paths of other separators for the remainder of their lengths. An example of this behavior is shown by the pink, orange, purple, and cyan separators in Figures 2(d), (b), and (c). Such behavior has been seen before in potential (Close et al. 2004) and non-potential fields (Haynes \& Parnell 2010).

Again we confirm the existence of our separators by comparing them with connectivity maps plotted on planes between the null clusters (Figures 2(b)-(d)). These show the four connectivities of flux present in the model as different colored regions that form a complex twirled pattern. Wherever there is a junction of the four flux domains a separator is found to be present. The insets Figures 2(b(i)) and (c(i)) show that if we zoom in then only one separator appears at each junction. Clearly, the boundary between the different flux domains is highly structured and appears to be interwoven, thus creating many separators. This occurs due to the fragmentation of the original current layer which was built up during the rise of the flux tube (Galsgaard et al. 2005). Such behavior has been observed before (e.g., Loureiro et al. 2007; Samtaney et al. 2009; Hood et al. 2009; Ishizawa \& Nakajima 2010). The exact instability that triggers the reconnection and the fragmentation of the current layer is not currently well understood and may differ depending on the particular model studied. It is, however, likely to be some form of resistive instability such as a tearing, gravitational, or a rippling mode. To determine which of these instabilities is dominant requires further work and will be discussed in more detail in a future paper.

Note that the connectivity maps reflect a global property, the connectivity, of the field lines that thread them and say nothing about the local behavior of the field in the vicinity of the planes of the maps. Thus, structures on scales at resolutions finer than the numerical grid may arise naturally in exactly the same way that fine scales are found in $Q$, the squashing factor (e.g., Titov et al. 2002; Aulanier et al. 2005; Restante et al. 2009).

\subsection{Importance of Separators for Reconnection}

Separators are first formed at the onset of reconnection and some of those in snapshot 86 are short-lived although others in snapshot 109 last for a significant fraction of the experiment. The current layer in which the main reconnection occurs develops once the emerging flux tube reaches the base of the overlying field at $50 \tau_{c s}$ before the reconnection starts at $53 \tau_{c s}$. For a current layer to form all that is needed is a strong gradient of the magnetic field which naturally occurs at the boundary between the two original flux domains (emerging flux tube and overlying field) and thus a current layer can exist in a system with two (or less) flux domains. By definition, however, separators cannot exist unless there are at least four flux domains. The only way a further two domains may be created here is by reconnection between the two original flux domains creating field lines that run from the flux tube to the corona and vice versa. Indeed, a separator is formed at the instant that reconnection starts (Haynes et al. 2007). Thus, it is not a coincidence that the arched mesh of inter-cluster separators threads the current layer which is associated with regions of high $E_{\|}$. The highly fragmentary nature of this reconnection explains the short lifetimes of the separators. This is because a separator will only last as long as it takes to reconnect the field trapped in its surrounding original flux domains. If one of these has only a small amount of flux then the separator will not last long, as noted by Haynes et al. (2007).

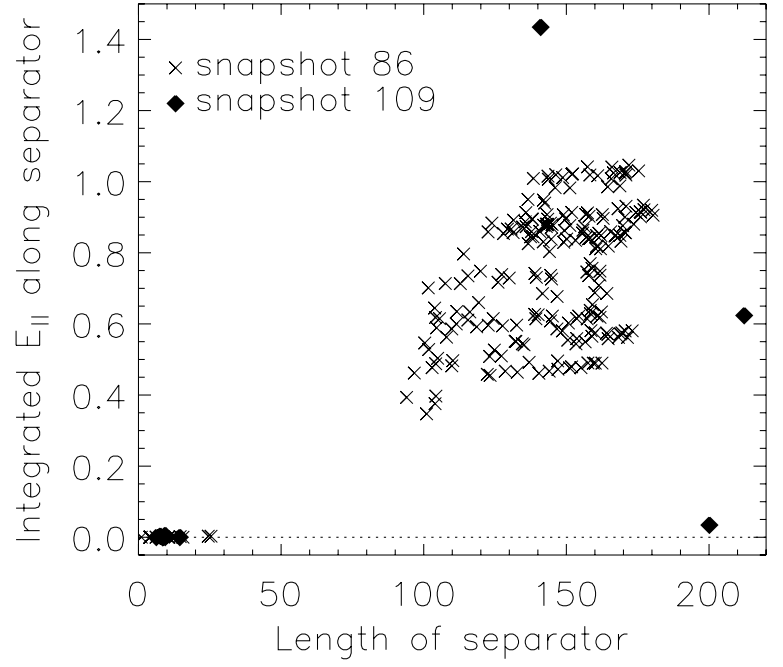

Figure 4. Plot of integrated $E_{\|}$along the separators vs. their lengths for all the separators in snapshot 1 (crosses) and snapshot 2 (diamonds).

We have shown evidence that the separators coincide with local maxima in $\int E_{\|} d l$. The larger the $\int E_{\|} d l$ the more significant the reconnection is at (and within the vicinity of) that separator. However, it is interesting to consider if all the separators are equally important for reconnection. In Figure 4, the $\int E_{\|} d l$ along the separators is plotted against separator length, for all the separators from both snapshots. As discussed earlier, a unit length equals $H_{\mathrm{ph}}=170 \mathrm{~km}$ and an $\int E_{\|} d l$ unit equals $c_{s} B_{\mathrm{ph}} H_{\mathrm{ph}}=150 \mathrm{~V}$. There is an obvious distinction between the short separators $(<30)$ joining nulls within each cluster which have $\int E_{\|} d l<10^{-3}$, while the long inter-cluster separators $(>90)$ have $\int E_{\|} d l>0.3$. It is clear though that the length of a separator is not necessarily proportional to its $\int E_{\|} d l$. Indeed, the longest separators in snapshot 109 which have lengths of 200 and 212 have lower $\int E_{\|} d l$ than most of the other inter-cluster separators in both snapshots which are all less than 185 long. This is not surprising since the two extra long inter-cluster separators both dip down below the photosphere and so they do not thread through the regions of strong $E_{\|}$ (Figure 1(a)).

There is no significant magnetic reconnection occurring along the separators within the null clusters and one of the very long inter-cluster separators. This can be understood by realizing that simply having field lines with different magnetic connectivities lying next to each other is not a sufficient condition for reconnection to take place. It is also necessary for there to be a plasma flow driving magnetic flux across the separators. Therefore, reconnection will not take place within the null clusters, but is strong at certain locations along the inter-cluster separators where the rising motion of the flux tube drives magnetic flux across the separators that divide it from the overlying field.

The nature of separator reconnection is discussed in detail in Parnell et al. (2010), who studied reconnection at separators in a different numerical MHD experiment. They found that separator reconnection does not just involve those field lines that go through the null points and lie along the separator, but it involves all field lines that penetrate the finite diffusion volumes through which the separators thread (Hornig \& Priest 2003; Priest et al. 2003). There may be just one long extended diffusion region or multiple regions of enhanced $E_{\|}$. In Figure 2(a), there appear to be several regions of enhanced $E_{\|}$along the separators. 
Such "hot spots" have been seen before by Parnell et al. (2010) and further work is required to determine the significance of these regions.

\section{DISCUSSION}

This work is a preliminary study of the separators found in two snapshots from a 3D numerical MHD model of solar flux emergence. Two hundred twenty-nine unique separators (emanating from 18 nulls) are found during the early complex interaction between the emerging flux tube and the overlying coronal field (snapshot 86). Eleven unique separators (emanating from 10 nulls) are found in snapshot 109 , which is taken from the later steady reconnection phase. The number of separators found in a snapshot bears no simple relationship to the number of nulls, but rather can only be understood by studying the previous 3D magnetic configuration and the plasma flows in the box. In both snapshots, the nulls are grouped in two loose clusters flanking the emerging flux tube. Magnetic reconnection sites are located along portions of the long separators that join the two null clusters with these separators sited at local maxima of $\int E_{\|} d l$. Not all of these separators are equally important for reconnection, but the reconnection along them is much more significant than along the short separators located wholly within the null clusters. Furthermore, outside the current layer no signatures of reconnection are detected in the numerical domain.

These results show that separator reconnection is an important and fundamental process for the interaction of emerging flux with overlying magnetic field. Reconnection takes place at multiple locations along the separators that form an arch over the emerging flux tube, joining the two null clusters. These separators define the junctions between four topologically distinct flux domains. Moreover, the rising motion of the flux tube drives magnetic flux across the separators, leading to significant magnetic reconnection. Since separator reconnection involves all field lines that penetrate the finite volumes of enhanced $E_{\|}$ around the long separators, a considerable amount of flux over a wide area can be reconnected at anyone instant. In future work, we will study the evolution of the separators and diffusion regions throughout the entire experiment, quantify how the magnetic reconnection varies in space and time, and determine the energy released through reconnection. Also, it would be interesting to compare our results with the complementary insights that may be gained from a geometric analysis of the magnetic field, i.e., calculating the locations and behavior of the quasi-separatrix layers (Priest \& Demoulin 1995; Démoulin et al. 1996) and quasi-separators/hyperbolic flux tubes (Titov et al. 2002, 2009).
The authors thank Dr. Klaus Galsgaard for providing the data analyzed and for his useful advice. They also thank the referee and Prof. Eric Priest for suggestions that have improved this manuscript. R.C.M. was funded by the St Andrew's solar group STFC rolling grant. A.L.H. and C.E.P. are grateful to the Leverhulme trust for funding via a Leverhulme prize.

\section{REFERENCES}

Archontis, V., Moreno Insertis, F., Galsgaard, K., \& Hood, A. W. 2005, ApJ, 635,1299

Archontis, V., Moreno Insertis, F., Galsgaard, K., Hood, A. W., \& O'Shea, E. 2004, A\&A, 426, 1047

Aulanier, G., Pariat, E., \& Demoulin, P. 2005, A\&A, 444, 961

Close, R. M., Parnell, C. E., \& Priest, E. R. 2004, Sol. Phys., 225, 21

Démoulin, P., Priest, E. R., \& Lonie, D. P. 1996, J. Geophys. Res., 101, 7631

Galsgaard, K., Archontis, V., Moreno Insertis, F., \& Hood, A. W. 2007, ApJ, 666,516

Galsgaard, K., Moreno Insertis, F., Archontis, V., \& Hood, A. W. 2005, ApJ, 618, L153

Galsgaard, K., \& Nordlund, A. 1997, J. Geophys. Res., 102, 231

Galsgaard, K., Priest, E. R., \& Nordlund, A. 2000, Sol. Phys., 193, 1

Green, J. M. 1989, J. Geophys. Res., 93, 8583

Haynes, A. L., \& Parnell, C. E. 2010, Phys. Plasmas, 17, 092903

Haynes, A. L., Parnell, C. E., Galsgaard, K., \& Priest, E. R. 2007, Proc. R. Soc A, 463, 1097

Hesse, M., \& Schindler, K. 1988, J. Geophys. Res., 93, 5559

Hood, A. W., Browning, P. K., \& Van der Linden, R. A. M. 2009, A\&A, 506 913

Hornig, G., \& Priest, E. R. 2003, Phys. Plasmas, 10, 2712

Ishizawa, A., \& Nakajima, N. 2010, Phys. Plasmas, 17, 072308

Lau, Y.-T., \& Finn, J. M. 1990, ApJ, 350, 672

Longcope, D. W. 2001, Phys. Plasmas, 8, 5277

Longcope, D. W. 2005, Living Rev. Sol. Phys., 2, 7

Longcope, D. W., \& Cowley, S. C. 1996, Phys. Plasmas, 3, 2885

Loureiro, N. F., Schekochihin, A. A., \& Cowley, S. C. 2007, Phys. Plasmas, 14, 100703

Maclean, R. C., Parnell, C. E., \& Galsgaard, K. 2009, Sol. Phys., 260, 299

Nordlund, A., \& Galsgaard, K. 1997, A 3D MHD Code for Parallel Computers (Tech. Rep.; Copenhagen: Astronomical Observatory, Copenhagen Univ.)

Parnell, C. E. 2007, Sol. Phys., 242, 21

Parnell, C. E., Haynes, A. L., \& Galsgaard, K. 2008, ApJ, 675, 1656

Parnell, C. E., Haynes, A. L., \& Galsgaard, K. 2010, J. Geophys. Res., 115, A02102

Pontin, D. I., \& Craig, I. J. D. 2006, ApJ, 642, 568

Priest, E. R., \& Demoulin, P. 1995, J. Geophys. Res., 100, 23443

Priest, E. R., Hornig, G., \& Pontin, D. I. 2003, J. Geophys. Res., 108, 1285

Priest, E. R., Longcope, D. W., \& Heyvaerts, J. 2005, ApJ, 624, 1057

Priest, E. R., \& Schrijver, C. J. 2004, Sol. Phys., 190, 1

Restante, A. L., Aulanier, G., \& Parnell, C. E. 2009, A\&A, 508, 433

Samtaney, R., Loureiro, N. F., Uzdensky, D. A., Schekochihin, A. A., \& Cowley, S. C. 2009, Phys. Rev. Lett., 103, 105004

Sonnerup, B. U. O. 1979, Magnetic Field Reconnection, in Space Plasma Physics: The Study of Solar-System Plasmas, Vol. 2 (Washington, DC: Natl. Acad. Press), 879

Titov, V. S., Forbes, T. G., Priest, E. R., Mikic, Z., \& Linker, J. A. 2009, ApJ, 693, 1029

Titov, V. S., Hornig, G., \& Demoulin, P. 2002, J. Geophys. Res., 107, 1164 